\title{
Strong approximations for long memory sequences based partial sums, counting and their Vervaat processes
}

\author{
Endre Csáki* \\ A. Rényi Institute of Mathematics, Hung. Acad. Sci. \\ Budapest, P.O.B. 127, H-1364 Hungary \\ e-mail: csaki.endre@renyi.mta.hu \\ Miklós Csörgő $†$ \\ School of Mathematics and Statistics, Carleton University \\ Ottawa, Ontario, Canada K1S 5B6 \\ e-mail: mcsorgo@math.carleton.ca \\ Rafał Kulik \\ Department of Mathematics and Statistics, University of Ottawa \\ Ottawa, Ontario, Canada K1N 6N5 \\ e-mail: rkulik@uottawa.ca
}

February 18, 2013

\begin{abstract}
We study the asymptotic behaviour of partial sums of long range dependent random variables and that of their counting process, together with an appropriately normalized integral process of the sum of these two processes, the so-called Vervaat process. The first two of these processes are approximated by an appropriately constructed fractional Brownian motion, while the Vervaat process in turn is approximated by the square of the same fractional Brownian motion.
\end{abstract}

Key words and phrases: Long range dependence, Linear process, Partial sums, Vervaat-type processes, Strong approximation, Fractional Brownian motion

\footnotetext{
${ }^{*}$ Research supported by the Hungarian National Foundation for Scientific Research No. K108615

${ }^{\dagger}$ Research supported by an NSERC Canada Discovery Grant at Carleton University, Ottawa

${ }^{\ddagger}$ Research supported by an NSERC Canada Discovery Grant at the University of Ottawa, Ottawa
} 
2000 Mathematics Subject Classification: Primary 60F15; Secondary 60F17 60G22

\section{Introduction}

Let $\left\{\tilde{\eta}_{j}, j \geq 0\right\}$ be a stationary long range dependent (LRD) centered Gaussian sequence with $\mathrm{E}\left(\tilde{\eta}_{0}^{2}\right)=1$ and covariance function of the form

$$
\rho_{k}:=\mathrm{E}\left(\tilde{\eta}_{0} \tilde{\eta}_{k}\right)=k^{-\alpha} L(k), \quad k=1,2, \ldots,
$$

where $\alpha \in(0,1)$, and $L(\cdot)$ is a slowly varying function at infinity. Let $G(\cdot)$ be a real valued Borel measurable function with $\mathrm{E}\left(G\left(\tilde{\eta}_{0}\right)\right)=\mu$ and $\mathrm{E}\left(G^{2}\left(\tilde{\eta}_{0}\right)\right)<\infty$. It may be expanded as

$$
G\left(\tilde{\eta}_{0}\right)-\mu=\sum_{q=m}^{\infty} \frac{J_{q}}{q !} H_{q}\left(\tilde{\eta}_{0}\right),
$$

where convergence is in $L^{2}$,

$$
H_{q}(x):=(-1)^{q} e^{x^{2} / 2} \frac{d^{q}}{d x^{q}} e^{-x^{2} / 2}, \quad q=1,2, \ldots,
$$

are Hermite polynomials, and $J_{q}:=\mathrm{E}\left(G\left(\tilde{\eta}_{0}\right) H_{q}\left(\tilde{\eta}_{0}\right)\right)$. The index $m$ in the above expansion is defined as

$$
m:=\min \left\{q \geq 1: J_{q} \neq 0\right\} .
$$

We then say that the Hermite rank of $G(\cdot)$ is $m$.

Consider the subordinated sequence $\left\{Y_{j}=G\left(\tilde{\eta}_{j}\right), j \geq 0\right\}$. Then $\mathrm{E}\left(Y_{j}\right)=\mu$, $j=0,1,2, \ldots$ Given (11) with $\alpha \in(0,1)$, assume that the Hermite rank $m$ of $G(\cdot)$ is such that $0<\alpha<1 / m$. Then, as $n \rightarrow \infty$, with $L(\cdot)$ as in (11), we have (cf. Lemma 3.1 and Theorem 3.1 of Taqqu [24], or [25]),

$$
\sigma_{n, m}^{2}:=\operatorname{Var}\left(\sum_{j=1}^{n}\left(Y_{j}-\mu\right)\right) \sim \frac{J_{m}^{2}}{m !} \frac{2}{(1-\alpha m)(2-\alpha m)} n^{2-m \alpha} L^{m}(n),
$$

where the symbol $\sim$ stands for the indicated terms being asymptotically equal to each other.

We note that an LRD Gaussian sequence as in (1) can be viewed in terms of the linear (moving average) process

$$
\eta_{j}=\sum_{k=0}^{\infty} \psi_{k} \xi_{j-k}, \quad j=0,1,2, \ldots
$$


where $\left\{\xi_{k},-\infty<k<\infty\right\}$ is a double sequence of independent standard normal random variables, and the sequence of weights $\left\{\psi_{k}, k=0,1,2, \ldots\right\}$ is square summable. Then $\mathrm{E}\left(\eta_{0}\right)=0, \mathrm{E}\left(\eta_{0}^{2}\right)=\sum_{k=0}^{\infty} \psi_{k}^{2}=: \sigma^{2}$ and, on putting $\tilde{\eta}_{j}=\eta_{j} / \sigma$, $\left\{\tilde{\eta}_{j}, j=0,1,2, \ldots\right\}$ is a stationary Gaussian sequence with $\mathrm{E}\left(\tilde{\eta}_{0}\right)=0$ and $\mathrm{E}\left(\tilde{\eta}_{0}^{2}\right)=1$. If $\psi_{k} \sim k^{-(1+\alpha) / 2} \ell(k)$ with a slowly varying function, $\ell(k)$, at infinity, then (cf. Wang et al. [29] $\mathrm{E}\left(\eta_{j} \eta_{j+n}\right) \sim b_{\alpha} n^{-\alpha} \ell^{2}(n)$, i.e., we have (11) with $L(n) \sim b_{\alpha} \ell^{2}(n) / \sigma^{2}$, where the constant $b_{\alpha}$ is defined by

$$
b_{\alpha}=\int_{0}^{\infty} x^{-(1+\alpha) / 2}(1+x)^{-(1+\alpha) / 2} d x .
$$

For more information on long memory Gaussian sequences and their functionals we refer to [24] and [26].

In this exposition we study the asymptotic behaviour of partial sums of long range dependent random variables and that of their counting process, together with an appropriately normalized integral process of the sum of these two processes, the so-called Vervaat process. All this will amount to an extension of the recent work in [2] and [3] to LRD sequences.

\subsection{Bahadur-Kiefer processes}

For initiating our discussion, let $U_{j}, j=1,2, \ldots$, be independent identically distributed uniform random variables on $[0,1]$, and denote by $F_{n}$ the right continuous empirical distribution function of the first $n \geq 1$ of these random variables. Define the so-called uniform Bahadur-Kiefer process $\left\{R_{n}(t), 0 \leq t \leq 1\right\}$ as

$$
R_{n}(t):=\alpha_{n}(t)+\beta_{n}(t)
$$

where $\alpha_{n}(t):=n^{1 / 2}\left(F_{n}(t)-t\right), \beta_{n}(t):=n^{1 / 2}\left(F_{n}^{-1}(t)-t\right)$, and $F_{n}^{-1}(\cdot)$ is the empirical quantile function (left continuous inverse of $F_{n}(\cdot)$ ). The process $R_{n}(\cdot)$ was introduced and first studied by Bahadur [1] and, consequently, by Kiefer in [13] and [14]. In particular, it can be concluded from the results of Kiefer in the just cited papers that the Bahadur-Kiefer process $R_{n}(\cdot)$ cannot converge weakly to any non-degenerate random element of the space $D[0,1]$. This can also be deduced via the following result of Vervaat in [28] and [27]: as $n \rightarrow \infty$,

$$
V_{n}(t):=2 n^{1 / 2} \int_{0}^{t} R_{n}(u) d u \Rightarrow W_{0}^{2}(t), \quad 0 \leq t \leq 1
$$

where $W_{0}(t)$ is a standard Brownian bridge, and $\Rightarrow$ stands for weak convergence in $C[0,1]$, equipped with the uniform norm. Since $W_{0}^{2}$ is not differentiable, we conclude again that $R_{n}$, as the derivative of $V_{n}$ cannot converge weakly in $D[0,1]$ 
with any normalization. The integrated Bahadur-Kiefer process in (5D) is usually referred to as the uniform Vervaat process.

The just mentioned works of Vervaat constitute important general contributions to limit theorems for processes with positive drift and their inverses which we now indicate briefly. Accordingly, let $\mathcal{Z}$ be a non-negative stochastic process on $[0, \infty)$ such that almost all realizations $\mathcal{Z}(\cdot, \omega):[0, \infty) \rightarrow[0, \infty)$ are nondecreasing unbounded functions, and $\mathcal{Z}^{-1}$ be the generalized inverse of $\mathcal{Z}(\cdot, \omega)$, i.e., $\mathcal{Z}^{-1}(t, \omega):=\inf \{u: \mathcal{Z}(u, \omega)>t\}$. Let $D_{0}[0, \infty)$ be the subset of non-decreasing, non-negative unbounded functions of $D[0, \infty)$, the set of real-valued functions on $[0, \infty)$ which are right-continuous and have finite left-hand limits at every point $t \in(0, \infty)$, and let $C[0, \infty)$ be the subset of continuous functions of $D[0, \infty)$. For further use we state here Theorem 1 of Vervaat [28] for our convenience as follows (cf. also Theorem 3.2.3 of Vervaat [27]). Here and throughout $\Rightarrow$ indicates weak convergence in an appropriate context, while $\stackrel{P}{\rightarrow}$ designates convergence in probability.

Theorem 1.1 Let $\mathcal{Z}_{1}, \mathcal{Z}_{2}, \ldots$, be random elements in $D_{0}[0, \infty), \tilde{\mathcal{Z}}$ a random element in $C[0, \infty)$, and $\zeta_{1}, \zeta_{2}, \ldots$, be positive random variables such that $\zeta_{n} \stackrel{P}{\rightarrow} 0$ as $n \rightarrow \infty$. Then, as $n \rightarrow \infty$, the following two weak convergence statements are equivalent in $D[0, \infty)$ (endowed with the uniform topology on compact sets):

$$
\begin{gathered}
\frac{\mathcal{Z}_{n}-I}{\zeta_{n}} \Rightarrow \tilde{\mathcal{Z}}, \\
\frac{\mathcal{Z}_{n}^{-1}-I}{\zeta_{n}} \Rightarrow-\tilde{\mathcal{Z}},
\end{gathered}
$$

where I denotes the identity map on $[0, \infty)$. Moreover, if any of the above statements holds, then

$$
V\left(\cdot ; \mathcal{Z}_{n}\right) \Rightarrow \frac{1}{2} \widetilde{\mathcal{Z}}^{2}
$$

in $C[0, \infty)$ (endowed with the uniform topology on compact sets), where

$$
V\left(t ; \mathcal{Z}_{n}\right)=\frac{1}{\zeta_{n}^{2}} \int_{0}^{t}\left(\mathcal{Z}_{n}(u)+\mathcal{Z}_{n}^{-1}(u)-2 u\right) d u, \quad 0 \leq t<\infty .
$$

Clearly, the statement of (5) is implied by that of (6).

On the other hand, if we consider a subordinated sequence of random variables $Y_{j}=G\left(\tilde{\eta}_{j}\right), j=0,1,2, \ldots$, with marginal distribution function $F(x)=P\left(Y_{0} \leq x\right)$, $x \in \mathbb{R}$, where $\left\{\tilde{\eta}_{j}, j \geq 0\right\}$ is a stationary LRD centered Gaussian sequence with $E\left(\tilde{\eta}_{0}^{2}\right)=1$ and covariance as in (1), then their appropriately scaled Bahadur-Kiefer process does converge weakly. To be more specific, on expanding $1\left\{Y_{0} \leq x\right\}-F(x)$ 
in terms of Hermite polynomials for any fixed $x \in \mathbb{R}$ as in Dehling and Taqqu [10], we have

$$
1\left\{Y_{0} \leq x\right\}-F(x)=\sum_{q=m_{x}}^{\infty} \frac{c_{q}(x)}{q !} H_{q}\left(\tilde{\eta}_{0}\right), \quad x \in \mathbb{R},
$$

where $c_{q}(x)=\mathrm{E}\left(1\left\{G\left(\tilde{\eta}_{0}\right) \leq x\right\}-F(x)\right) H_{q}\left(\tilde{\eta}_{0}\right)$, and $m_{x}$ for any $x \in \mathbb{R}$ is the index of the first nonzero coefficient in this expansion, the so-called Hermite rank of $1\left\{Y_{0} \leq x\right\}-F(x)$. Then, the Hermite rank of the class $\left(1\left\{Y_{0} \leq x\right\}-F(x), x \in \mathbb{R}\right)$ is defined as

$$
m:=\min \left\{m_{x}: c_{m_{x}}(x) \neq 0 \quad \text { for some } x \in \mathbb{R}\right\} .
$$

On assuming now that $F(\cdot)$ is continuous, in terms of the first $n \geq 1$ of the uniformly distributed random variables $U_{j}=F\left(Y_{j}\right)=F\left(G\left(\tilde{\eta}_{j}\right)\right), j=1,2, \ldots$, keeping the notation we already used in the i.i.d. case in (44) for the LRD case as well, we redefine $\alpha_{n}(\cdot)$ and $\beta_{n}(\cdot)$, respectively, as

$$
\begin{array}{ll}
\alpha_{n}(t):=\frac{n}{d_{n, m}}\left(F_{n}(t)-t\right), & t \in[0,1], \\
\beta_{n}(t):=\frac{n}{d_{n, m}}\left(F_{n}^{-1}(t)-t\right), & t \in[0,1],
\end{array}
$$

where $d_{n, m}^{2}=n^{2-m \alpha} L^{m}(n)$.

In this context, from Theorem 1.1 of Dehling and Taqqu [10, we obtain that as long as $0<\alpha<1 / m$, as $n \rightarrow \infty$,

$$
\alpha_{n}(t) \Rightarrow \sqrt{\frac{2}{(2-m \alpha)(1-m \alpha)}} c_{m}\left(F^{-1}(t)\right) X_{m},
$$

in $D[0,1]$, equipped with the sup-norm, where $F^{-1}(\cdot)$ is the quantile function (inverse) of $F(\cdot), X_{m} \stackrel{\mathrm{d}}{=} X_{m}(1)$, where $\left\{X_{m}(s), 0 \leq s \leq 1\right\}$ is $1 / m$ ! times a Hermite process of rank $m$, given for each $s$ as a multiple Wiener-Itô-Dobrushin integral (see, e.g., [10], 26]).

As a consequence of (8) and Theorem 1.1, we immediately conclude also that, as $n \rightarrow \infty$,

$$
\beta_{n}(t) \Rightarrow-\sqrt{\frac{2}{(2-m \alpha)(1-m \alpha)}} c_{m}\left(F^{-1}(t)\right) X_{m},
$$

in $D[0,1]$, equipped with the sup-norm.

Furthermore, on using the same definition of the Bahadur-Kiefer process as in (44) in our present LRD context as well, via (6) we arrive at

$$
\frac{n}{d_{n, m}} \int_{0}^{t} R_{n}(u) d u \Rightarrow \frac{1}{(2-m \alpha)(1-m \alpha)} c_{m}^{2}\left(F^{-1}(t)\right) X_{m}^{2},
$$


in $C[0,1]$, endowed with the uniform topology.

Now, unlike in the i.i.d. case (cf. (5)), we can formally differentiate the processes in (9), and thus obtain the following corollary.

Corollary 1.2 Let $0<\alpha<1 / m$, where $m$ is as in (7), and let $X_{m}$ be the random variable defined in (8). Assume that the marginal distribution function $F$ has a positive density $f=F^{\prime}$, with respect to Lebesgue measure, on $(a, b)$, where $a=$ $\sup \{x: F(x)=0\}, b=\inf \{x: F(x)=1\},-\infty \leq a<b \leq \infty$. Assume that the function $c_{m}(\cdot)$ is also differentiable over this interval $(a, b)$. Then, as $n \rightarrow \infty$,

$$
\frac{n}{d_{n, m}} R_{n}(t) \Rightarrow \frac{2}{(2-m \alpha)(1-m \alpha)} \frac{c_{m}\left(F^{-1}(t)\right) c_{m}^{\prime}\left(F^{-1}(t)\right)}{f\left(F^{-1}(t)\right)} X_{m}^{2},
$$

in $D[0,1]$, endowed with the sup-norm, provided the deterministic function

$$
\frac{c_{m}\left(F^{-1}(t)\right) c_{m}^{\prime}\left(F^{-1}(t)\right)}{f\left(F^{-1}(t)\right)}
$$

is finite for $t \in[0,1]$.

Thus, unlike in the i.i.d. case, the LRD-based Bahadur-Kiefer process does converge weakly. Dealing with the sequential version of (10), this was first observed in [8]; see also [9], 4], [5]. In these papers the method of establishing (10) was in fact completely different. Namely, strong approximations were used instead of making use of Vervaat's approach in hand. This, however, led to stronger assumptions on $F$. Nevertheless, the method of strong approximations as in the above mentioned works is needed to deal with weak and strong laws of the general (non-uniform) and possibly sequential Bahadur-Kiefer and Vervaat processes that are based on subordinated sequences or linear processes.

\subsection{Partial Sums and Counting Processes}

We are to see in this exposition that, unlike Bahadur-Kiefer processes, i.i.d. and LRD based sums of partial sums and their counting processes behave similarly in that both cannot converge weakly.

Specifically now, assume that $\mathrm{E}\left(Y_{0}\right)=\mu$, put $S_{n}:=Y_{1}+\cdots+Y_{n}, n \geq 1$, and define, as in [3],

$$
S_{n}(t):=(n \mu)^{-1} S_{[n t]}, \quad N_{n}(t):=N(n \mu t) / n,
$$

where $N(t):=\min \left\{n \geq 1: S_{n}>t\right\}$.

Assume first that the random variables $Y_{j}$ are i.i.d., and, via $S_{n}(\cdot)$ and $N_{n}(\cdot)$ as in (11) define now the following analogue of the Bahadur-Kiefer process $R_{n}$ as in (4) as follows:

$$
R_{n}^{*}(s):=n^{1 / 2}\left(S_{n}(s)-s+N_{n}(s)-s\right)
$$


Then, in view of the left-hand side of the statement in (5), we define the following Vervaat-type process:

$$
\tilde{V}_{n}(t):=n \int_{0}^{t}\left\{\left(S_{n}(s)-s\right)+\left(N_{n}(s)-s\right)\right\} d s=n^{1 / 2} \int_{0}^{t} R_{n}^{*}(s) d s .
$$

In [3] strong approximations were established for $V_{n}(\cdot)$ in terms of the square of a standard Brownian motion under the conditions $P\left(Y_{0}>0\right)=1$ and $\mathrm{E}\left(Y_{0}^{4}\right)<\infty$.

In 2] the condition $\mathrm{E}\left(Y_{0}^{4}\right)<\infty$ was replaced by a weaker moment condition and the results of [3] were extended to weakly dependent cases.

In view of Donsker's theorem for partial sums of i.i.d. random variables, it follows from Theorem 1.1 that, if $P\left(Y_{0} \geq 0\right)=1$ and $0<\mathrm{E}\left(Y_{0}^{2}\right)<\infty$, the i.i.d. based Vervaat-type process $\left\{\tilde{V}_{n}(t), t \in[0,1]\right\}$ converges weakly in $C[0,1]$ (equipped with the uniform topology) to $\left\{W^{2}(t), t \in[0,1]\right\}$, where the latter process is the square of a standard Brownian motion. Consequently, similarly to (5), the "derivative" of $\tilde{V}_{n}(t)$, i.e., the i.i.d. based integrand process $\left\{R_{n}^{*}(t), t \in[0,1]\right\}$ as above cannot converge weakly.

It will be shown in this paper that in case of LRD sequences, for $R_{n}^{*}(\cdot)$ we have a similar lack of weak convergence. Namely, a strong approximation of the appropriately scaled LRD sequences based $\left\{\tilde{V}_{n}(t), t \in[0,1]\right\}$ yields a limiting process in terms of the square of a fractional Brownian motion. Consequently, its LRD based integrand process $\left\{R_{n}^{*}(t), t \in[0,1]\right\}$ cannot converge weakly. This is in contrast to having the weak convergence in the LRD case of the appropriately scaled BahadurKiefer process $R_{n}(\cdot)$ as in (10).

In order to conclude our strong approximation for an LRD sequences based Vervaat process, we first establish some new results on the almost sure behaviour of similarly based partial sums and their counting process.

The paper is organized as follows. In Section 2.1, we introduce our basic assumptions and notations for the sake of stating our strong approximation results for LRD based partial sums, their counting process and the therein defined Vervaat process. In Section 2.2, we list weak convergence laws and laws of the iterated logarithm (LIL) for these LRD based processes via well-known laws for appropriate fractional Brownian motions. The proofs are given in Section 3. In particular, in Section 3.1. we first describe the construction of the fractional Brownian motion that will be used throughout later on when proving our strong approximation results for our LRD sequences based processes in hand. The latter approximations will be achieved by an appropriate reduction principle and strong approximation for partial sums of LRD based subordinated sequences that, in turn, conclude Section 3.1. Following this, in Sections 3.2 and 3.3 respectively, we establish our strong approximations for the counting and Vervaat processes in hand. 


\section{Results}

\section{$2.1 \quad$ Statements of results}

Put $\tilde{\eta}_{j}=\eta_{j} / \sigma, j=0,1,2, \ldots$, where $\eta_{j}$ is as in (3) with $\sigma^{2}=\mathrm{E}\left(\eta_{0}^{2}\right)=\sum_{k=0}^{\infty} \psi_{k}^{2}$. We shall assume for simplicity that the slowly varying function in (1) is constant, equal to one. We are now concerned with the subordinated sequence $Y_{j}=G\left(\tilde{\eta}_{j}\right)$, $j=0,1,2, \ldots$ We assume throughout that $J_{1}:=\mathrm{E}\left(G\left(\tilde{\eta}_{0}\right) \tilde{\eta}_{0}\right) \neq 0$, i.e., the Hermite rank of $G(\cdot)$ is assumed to be 1 .

We will make use of, or a part of, the following assumptions:

(i) $\mathrm{E} Y_{0}=\mu>0$;

(ii) $\mathrm{E}\left(Y_{0}^{2}\right)<\infty$;

(iii) $P\left(Y_{0} \geq 0\right)=1$.

In terms of our subordinated sequence $\left\{Y_{j}, j=0,1,2, \ldots\right\}$, with $t \geq 0$, we define

$$
\begin{aligned}
S(t) & :=\sum_{i=1}^{[t]} Y_{i}, \\
N(t) & :=\inf \{s \geq 1: S(s)>t\}, \\
Q(t) & :=S(t)+\mu N(\mu t)-2 \mu t, \\
Z(t) & :=\mu \int_{0}^{t} Q(s) d s .
\end{aligned}
$$

In terms of these definitions we have the following analogue of the BahadurKiefer process

$$
R_{n}^{*}(s):=\frac{S(n s)+\mu N(\mu n s)-2 \mu n s}{n^{1-\alpha / 2}}=n^{\alpha / 2} \frac{Q(n s)}{n} .
$$

Via $Z(\cdot)$, the Vervaat process in this context is defined by

$$
V_{n}(t):=n^{\alpha / 2} \int_{0}^{t} R_{n}^{*}(u) d u=n^{\alpha / 2} \int_{0}^{t} n^{\alpha / 2} \frac{Q(n u)}{n} d u=\frac{Z(n t)}{\mu n^{2-\alpha}} .
$$

For $1 / 2<H<1$ let $\left\{W_{H}(t), t \geq 0\right\}$ be a fractional Brownian motion, i.e. a mean-zero stationary Gaussian process with covariance

$$
\mathrm{E} W_{H}(s) W_{H}(t)=\frac{1}{2}\left(s^{2 H}+t^{2 H}-|s-t|^{2 H}\right) .
$$


Theorem 2.1 Let $\eta_{j}$ be defined by (3) with $\psi_{k} \sim k^{-(1+\alpha) / 2}, 0<\alpha<1$, and put $\tilde{\eta}_{j}=\eta_{j} / \sigma$ with $\sigma^{2}:=\mathrm{E}\left(\eta_{0}^{2}\right)=\sum_{k=0}^{\infty} \psi_{k}^{2}$. Let $G(\cdot)$ be a function whose Hermite rank is 1. Furthermore, let $\{S(t), t \geq 0\}$ be as in (12) and assume condition (ii). Then, on an appropriate probability space for the sequence $\left\{Y_{j}=G\left(\tilde{\eta}_{j}\right), j=0,1, \ldots\right\}$, one can construct a fractional Brownian motion $W_{1-\alpha / 2}(\cdot)$ such that, as $T \rightarrow \infty$, we have

$$
\sup _{0 \leq t \leq T}\left|S(t)-\mu t-\frac{J_{1} \kappa_{\alpha}}{\sigma} W_{1-\alpha / 2}(t)\right|=o_{\text {a.s. }}\left(T^{\gamma / 2+\delta}\right)
$$

where

$$
\kappa_{\alpha}^{2}=2 \frac{\int_{0}^{\infty} x^{-(\alpha+1) / 2}(1+x)^{-(\alpha+1) / 2} d x}{(1-\alpha)(2-\alpha)},
$$

$\gamma=2-2 \alpha$ for $\alpha<1 / 2, \gamma=1$ for $\alpha \geq 1 / 2$ and $\delta>0$ is arbitrary.

Theorem 2.2 Assume the conditions of Theorem 2.1 and condition (i). Then, on the probability space of Theorem 2.1 for the sequence $\left\{Y_{j}=G\left(\tilde{\eta}_{j}\right), j=0,1, \ldots\right\}$, together with the fractional Brownian motion $W_{1-\alpha / 2}(\cdot)$ as in (19), for $N(t)$ as in (13), as $T \rightarrow \infty$, we have

$$
\sup _{0 \leq t \leq T}\left|\mu N(\mu t)-\mu t+\frac{J_{1} \kappa_{\alpha}}{\sigma} W_{1-\alpha / 2}(t)\right|=o_{\text {a.s. }}\left(T^{\gamma / 2+\delta}+T^{(1-\alpha / 2)^{2}+\delta}\right)
$$

with $\gamma$ as in Theorem 2.1, and arbitrary $\delta>0$.

Theorem 2.3 Assume the conditions of Theorem 2.1 and condition (iii). Then, on the probability space of Theorem 2.1 for the sequence $\left\{Y_{j}=G\left(\tilde{\eta}_{j}\right), j=0,1, \ldots\right\}$, together with the fractional Brownian motion $W_{1-\alpha / 2}(\cdot)$ as in (19) and (21), for $Z(t)$ as in (15), as $T \rightarrow \infty$, we have

$$
\sup _{0 \leq t \leq T}\left|Z(t)-\frac{1}{2}\left(\frac{J_{1} \kappa_{\alpha}}{\sigma} W_{1-\alpha / 2}(t)\right)^{2}\right|=o_{\text {a.s. }}\left(T^{2-3 \alpha / 2+\alpha^{2} / 4+\delta}+T^{(1-\alpha / 2+\gamma / 2+\delta}\right)
$$

with $\gamma$ as in Theorem 2.1, and arbitrary $\delta>0$.

\subsection{Consequences of Theorems 2.12 .3}

First we deal with some immediate consequences of Theorem 2.1 for the partial sum process $S(t)$ as in (12).

Corollary 2.4 Under the conditions of Theorem [2.1, as $n \rightarrow \infty$, we have the following weak convergence in $D[0, \infty)$ (endowed with the uniform topology on compact sets).

$$
\frac{\sigma(S(n t)-\mu n t)}{J_{1} \kappa_{\alpha} n^{1-\alpha / 2}} \Rightarrow W_{1-\alpha / 2}(t) .
$$


We note in passing that a more general first version of this result was established directly on $D[0,1]$ by Taqqu [24].

Corollary 2.5 Under the conditions of Theorem 2.1, we have

$$
\begin{gathered}
\limsup _{n \rightarrow \infty} \frac{\sigma \sup _{0 \leq t \leq 1}|S(n t)-\mu n t|}{J_{1} \kappa_{\alpha} n^{1-\alpha / 2}(2 \log \log n)^{1 / 2}}=1 \quad \text { a.s. } \\
\liminf _{n \rightarrow \infty} \frac{\sigma \sup _{0 \leq t \leq 1}|S(n t)-\mu n t|}{J_{1} \kappa_{\alpha} n^{1-\alpha / 2}(\log \log n)^{-1+\alpha / 2}}=c_{\alpha} \quad \text { a.s., }
\end{gathered}
$$

where $c_{\alpha}$ is a positive constant.

Corollary 2.6 Let $a_{T}$ be a nondecreasing function of $T$ such that $T^{\tau+\delta}<a_{T} \leq T$ and $a_{T} / T$ is nonincreasing, where $\delta>0$ is arbitrary, $\tau=(2-2 \alpha) /(2-\alpha)$ if $0<\alpha<1 / 2$ and $\tau=1 /(2-\alpha)$ if $1 / 2 \leq \alpha<1$. Then, under the conditions of Theorem 2.1, we have

$$
\limsup _{T \rightarrow \infty} \frac{\sigma \sup _{0 \leq t \leq T-a_{T}} \sup _{0 \leq s \leq a_{T}}|S(t+s)-S(t)-\mu s|}{J_{1} \kappa_{\alpha} a_{T}^{1-\alpha / 2}\left(2\left(\log T / a_{T}+\log \log T\right)\right)^{1 / 2}}=1 \quad \text { a.s. }
$$

Remark 2.7 As to the result of (24), we note that, viewed via Theorem 2.1, it is inherited from the LIL for fractional Brownian motion that was established by Taqqu [25, cf. Corollary A1, in a more general functional form. The latter can also be spelled out for our $S(t)$ as in (12), via Theorem 2.1. Taqqu [25] establishes his functional LIL for partial sums like our (12) and Gaussian processes as in his Theorem A1 separetely, on their own. We note in passing that the statement of (21) also follows from the LIL for Gaussian processes of Theorem 1.1 of Orey [22].

Remark 2.8 The result in (25) is new for our partial sums, and it is inherited from (3.6) of Theorem 3.3 of Monrad and Rootzén [19], where it is concluded for a standard fractional Brownian motion, with index $1-\alpha / 2$ in our terms. Thus the latter version of Chung's law of the iterated logarithm for fractional Brownian motion is shared by partial sums of LRD sequences of random variables. As to the constant $c_{\alpha}$, its numerical value is not determined in [19].

Remark 2.9 Ortega [23] extended the large increment results of Csörgö-Révész [6], 7] for a Wiener process to centered Gaussian processes with stationary increments. Theorem 3 of 23 for a fractional Brownian motion as in (18) reads as follows: For $T>0$ let $a_{T}$ be a nondecreasing function of $T$ such that $0<a_{T} \leq T$ and $a_{T} / T$ is nonincreasing. Then

$$
\limsup _{T \rightarrow \infty} \frac{\sup _{0 \leq t \leq T-a_{T}} \sup _{0 \leq s \leq a_{T}}\left|W_{1-\alpha / 2}(t+s)-W_{1-\alpha / 2}(t)\right|}{a_{T}^{1-\alpha / 2}\left(2\left(\log T / a_{T}+\log \log T\right)\right)^{1 / 2}}=1 \quad \text { a.s. }
$$


Consequently, via Theorem 2.1, we arrive at (26). On taking $a_{T}=T$, we may conclude (24) with $T$ instead of $n$.

We continue with spelling out new results for the counting process $N(t)$ as in (13) that follow from Theorem 2.2.

Corollary 2.10 Under the conditions of Theorem 2.2, as $n \rightarrow \infty$, we have the following weak convergence in $D[0, \infty)$ (endowed with the uniform topology on compact sets).

$$
\frac{\sigma(\mu N(\mu n t)-\mu n t)}{J_{1} \kappa_{\alpha} n^{1-\alpha / 2}} \Rightarrow-W_{1-\alpha / 2}(t) .
$$

Corollary 2.11 Under the conditions of Theorem 2.2, we have

$$
\begin{gathered}
\limsup _{n \rightarrow \infty} \frac{\sigma \sup _{0 \leq t \leq 1}|\mu N(\mu n t)-\mu n t|}{J_{1} \kappa_{\alpha} n^{1-\alpha / 2}(2 \log \log n)^{1 / 2}}=1 \quad \text { a.s. } \\
\liminf _{n \rightarrow \infty} \frac{\sigma \sup _{0 \leq t \leq 1}|\mu N(\mu n t)-\mu n t|}{J_{1} \kappa_{\alpha} n^{1-\alpha / 2}(\log \log n)^{-1+\alpha / 2}}=c_{\alpha} \quad \text { a.s., }
\end{gathered}
$$

where the positive constant $c_{\alpha}$ is that of (25).

Corollary 2.12 Let $a_{T}$ be a nondecreasing function of $T$ such that $T^{\tau+\delta}<a_{T} \leq T$ and $a_{T} / T$ is nonincreasing, where $\delta>0$ is arbitrary, $\tau=(2-2 \alpha) /(2-\alpha)$ if $0<\alpha<1 / 2$ and $\tau=1 /(2-\alpha)$ if $1 / 2 \leq \alpha<1$. Then, under the conditions of Theorem 2.2, we have

$$
\limsup _{T \rightarrow \infty} \frac{\sigma \sup _{0 \leq t \leq T-a_{T}} \sup _{0 \leq s \leq a_{T}}|\mu(N(\mu t+\mu s)-N(\mu t))-\mu s|}{J_{1} \kappa_{\alpha} a_{T}^{1-\alpha / 2}\left(2\left(\log T / a_{T}+\log \log T\right)\right)^{1 / 2}}=1 \quad \text { a.s. }
$$

Remark 2.13 We note that, mutatis mutandis, the conclusions of Corollaries 2.10, 2.11, 2.12 for the counting process $N(t)$ follow via Theorem 2.2 exactly the same way as those of Corollaries 2.4, 2.5, 2.6 do for $S(t)$ from Theorem 2.1] as noted in Remarks 2.7, 2.8, 2.9, i.e., from known results for the fractional Brownian motion $W_{1-\alpha / 2}(t)$.

The next corollaries deal with the process $Z(\cdot)$ as in (15) or, equivalently, with the Vervaat process $V_{n}(\cdot)$ as in (17) via the strong approximation as in Theorem 2.3, in combination with known results for the fractional Brownian motion $W_{1-\alpha / 2}(t)$.

Corollary 2.14 Under the conditions of Theorem 2.3, as $n \rightarrow \infty$, we have the following weak convergence in $C[0, \infty)$ (endowed with the uniform topology on compact sets).

$$
\frac{2 \sigma^{2} Z(n t)}{J_{1}^{2} \kappa_{\alpha}^{2} n^{2-\alpha}}=\frac{2 \mu \sigma^{2} V_{n}(t)}{J_{1}^{2} \kappa_{\alpha}^{2}} \Rightarrow W_{1-\alpha / 2}^{2}(t)
$$


Corollary 2.15 Under the conditions of Theorem 2.3, we have

$$
\begin{array}{r}
\limsup _{n \rightarrow \infty} \frac{\sigma^{2} \sup _{0 \leq t \leq 1} Z(n t)}{J_{1}^{2} \kappa_{\alpha}^{2} n^{2-\alpha} \log \log n}=\limsup _{n \rightarrow \infty} \frac{\mu \sigma^{2} \sup _{0 \leq t \leq 1} V_{n}(t)}{J_{1}^{2} \kappa_{\alpha}^{2} \log \log n}=1 \quad \text { a.s. } \\
\liminf _{n \rightarrow \infty} \frac{2 \sigma^{2} \sup _{0 \leq t \leq 1} Z(n t)}{J_{1}^{2} \kappa_{\alpha}^{2} n^{2-\alpha}(\log \log n)^{-2+\alpha}}=\liminf _{n \rightarrow \infty} \frac{2 \mu \sigma^{2} \sup _{0 \leq t \leq 1} V_{n}(t)}{J_{1}^{2} \kappa_{\alpha}^{2}(\log \log n)^{-2+\alpha}}=c_{\alpha}^{2} \quad \text { a.s., }
\end{array}
$$

where the positive constant $c_{\alpha}$ is as in (25).

Remark 2.16 If we were to assume the conditions of Theorem 2.3 to begin with, then, as noted already, we would have (23) directly via Taqqu [24] that, in turn, in view of Theorem 1.1, would lead to having (28) and (32) as well, as a consequence of (23). Naturally, the respective strong approximation results of Theorems 2.1. 2.2, 2.3 were needed in order to conclude the strong laws of Corollaries 2.5, 2.11, 2.15 respectively. We also note in passing that the strong approximation result of Theorem 2.2 leads to the weak convergence conclusion of Corollary 2.10, on assuming only condition (i) instead of condition (iii), that is needed in the context of the first sentence above.

Remark 2.17 We note in passing that our positivity condition (iii) is used only in Theorem 2.3 in terms of our subordinated sequences $Y_{j}=G\left(\tilde{\eta}_{j}\right)$, for which an arbitrary marginal distribution function $F$ can always be obtained via choosing $G(\cdot)=F^{-1}(\Phi(\cdot))$, where $\Phi(\cdot)$ stands for the standard normal distribution function.

Remark 2.18 It would be of interest to prove analogues of our results at least for functions $G$ whose Hermite rank is $m=2$. This would give rise to the so-called Rosenblatt process (cf. Taqqu [24]). The latter process has stationary increments and covariance structure like that of a fractional Brownian motion in (18). However, it is non-Gaussian. Hence, difficulties arise when trying to deal with its path behaviour, though one would think that it should be similar to that of a fractional Brownian motion.

\section{Proofs}

To begin with, we note that the proof of Theorem 2.3, that is based on Theorems 2.1 and 2.2, also makes fundamental use of the basic algebraic identity that is (2.5) of [3]. In the i.i.d. case, the appropriate approximations follow from the KomlósMajor-Tusnády results [15, [16] and the work of Horváth [11]. 


\subsection{Preliminary results and proof of Theorem 2.1}

First we note that, via the proof of Lemma 6 in Oodaira [21], we borrow the following construction (cf. page 379 of Wang et al. 29]) for our fractional Brownian motion $W_{1-\alpha / 2}$ that is being used throughout this paper. We also note in passing that in the first line of page 379 in [29] one should write $\kappa_{\alpha}^{2}$ instead of $\kappa_{\alpha}$.

Lemma 3.1 Let $\eta_{j}$ be defined by (3) with $\psi_{k} \sim k^{-(1+\alpha) / 2}, 0<\alpha<1$, and put $\tilde{\eta}_{j}=\eta_{j} / \sigma$ with $\sigma^{2}:=\mathrm{E}\left(\eta_{0}^{2}\right)=\sum_{k=0}^{\infty} \psi_{k}^{2}$. Then, on an appropriate probability space for the sequence $\left\{\tilde{\eta}_{j}, j=0,1, \ldots\right\}$, one can construct a fractional Brownian motion $W_{1-\alpha / 2}(\cdot)$ such that, as $T \rightarrow \infty$, we have

$$
\sup _{0 \leq t \leq T}\left|\kappa_{\alpha}^{-1} \sigma \sum_{j=1}^{[t]} \tilde{\eta}_{j}-W_{1-\alpha / 2}(t)\right|=o_{\text {a.s. }}\left(T^{(1-\alpha) / 2} \log T\right),
$$

where $\kappa_{\alpha}$ is as in (20).

We now state and prove a lemma, a strong reduction principle in terms of a function with arbitrary Hermite rank. Earlier versions were given by Taqqu [25] and Kôno [17]. The present version is of a better rate that is based on combining a result of Lai and Stout [18] with that of Taqqu [25].

Lemma 3.2 Assume that $H(\cdot)$ is an arbitrary function such that $\mathrm{E}\left(H\left(\tilde{\eta}_{0}\right)\right)=0$, $\mathrm{E}\left(H^{2}\left(\tilde{\eta}_{0}\right)\right)<\infty$, and its Hermite rank is $m \geq 1$, where $\tilde{\eta}_{0}$ is a standard normal random variable. Let $\left\{\tilde{\eta}_{j}, j=0,1, \ldots\right\}$ be a stationary Gaussian sequence with correlation as in (1). Then, as $n \rightarrow \infty$,

$$
\sup _{0 \leq t \leq n}\left|\sum_{j=1}^{[t]} H\left(\tilde{\eta}_{j}\right)-\frac{J_{m}}{m !} \sum_{j=1}^{[t]} H_{m}\left(\eta_{j}\right)\right|=o_{\text {a.s. }}\left(n^{\gamma / 2+\delta}\right),
$$

where $\gamma=2-(m+1) \alpha$ for $\alpha<1 /(m+1), \gamma=1$ for $\alpha \geq 1 /(m+1)$ and $\delta>0$ is arbitrary.

Proof. Let

$$
U(n)=\sum_{j=1}^{n} H\left(\tilde{\eta}_{j}\right)-\frac{J_{m}}{m !} \sum_{j=1}^{n} H_{m}\left(\tilde{\eta}_{j}\right) .
$$

The Hermite rank of $H(\cdot)-J_{m} / m ! H_{m}(\cdot)$ is at least $m+1$. By Taqqu 25, Proposition $4.2]$ with $p=2$, we have for all $a \geq 0$

$$
\mathrm{E}\left((U(n+a)-U(a))^{2}\right) \leq C\left\{n \sum_{i=0}^{n}\left|\rho_{i}\right|^{m+1}\right\} \leq C n^{\gamma+\delta},
$$


where $C$ is a finite positive constant, $\gamma$ is as above and $\delta>0$ arbitrary. Consequently,

$$
P(|U(a+n)-U(a)| \geq x) \leq x^{-2} \mathrm{E}(U(a+n)-U(a))^{2} \leq x^{-2} n^{\gamma+\delta}
$$

for $x>0, a \geq 0, n \geq 1$.

Since $\gamma+\delta>1$, the conditions of Theorem 7 of Lai and Stout [18] are satisfied with $g(n)=n^{\gamma+\delta}$, and $p=2$. Hence, in our special case, with $\delta>0$ and $\gamma$ as in Theorem 2.1, we conclude

$$
\lim _{n \rightarrow \infty} \frac{U(n)}{\left(n^{\gamma+\delta}(\log n)^{1+\delta}\right)^{1 / 2}}=0, \quad \text { a.s. }
$$

Hence, as $n \rightarrow \infty$

$$
U(n)=o_{\text {a.s. }}\left(n^{\gamma / 2+\delta}\right)
$$

that, in turn, also yields (36) of Lemma 3.2.

Proof of Theorem 2.1. From Lemma 3.2 we obtain that, under the assumptions of Theorem 2.1, as $T \rightarrow \infty$, we have the following rate in the reduction principle for $\left\{Y_{j}=G\left(\tilde{\eta}_{j}\right) ; j=1,2, \ldots\right\}$ :

$$
\sup _{0 \leq t \leq T}\left|\sum_{j=1}^{[t]}\left(G\left(\tilde{\eta}_{j}\right)-\mu\right)-J_{1} \sum_{j=1}^{[t]} \tilde{\eta}_{j}\right|=o_{\text {a.s. }}\left(T^{\gamma / 2+\delta}\right),
$$

Since $1-\alpha<\gamma$, from (35) and (37) we conclude that, as $T \rightarrow \infty$,

$$
\sup _{0 \leq t \leq T}\left|S(t)-\mu t-\frac{J_{1} \kappa_{\alpha}}{\sigma} W_{1-\alpha / 2}(t)\right|=o_{\text {a.s. }}\left(T^{\gamma / 2+\delta}\right) .
$$

\subsection{Proof of Theorem 2.2}

Assume the conditions of Theorem 2.2, i.e., those of Theorem 2.1 and condition (i).

We follow the approach of Horváth [11], [12]. We first show that, as $T \rightarrow \infty$,

$$
\sup _{0 \leq t \leq T}|N(\mu t)-t|=o_{\text {a.s. }}(T) \text {. }
$$


Put $n=N(\mu t)$. Then $S(n) \leq \mu t \leq S(n+1)$. But according to (19) we have

$$
|S(n)-\mu n|=o_{\text {a.s. }}(n), \quad|S(n+1)-\mu n|=o_{\text {a.s. }}(n),
$$

from which

$$
|\mu t-\mu n|=o_{\text {a.s. }}(n)
$$

i.e.

$$
t=N(\mu t)+o_{\text {a.s. }}(N(\mu t)),
$$

which, in turn, implies (39).

Let $\Lambda(t)$ and $\lambda(t)$ be two functions on $t \in[0, \infty)$ such that $\Lambda(u)=\inf \{t \geq 0$ : $\lambda(t)>u\}$. Then (see, e.g., [11])

$$
\sup _{0<u<T}|\Lambda(u)-u| \leq \sup _{0<t<\Lambda(T)}|\lambda(t)-t| .
$$

Consequently, as $T \rightarrow \infty$, (39) in combination with (24) yields

$$
\begin{aligned}
& \sup _{0 \leq t \leq T}|N(\mu t)-t| \leq \sup _{0 \leq t \leq N(\mu T)}|S(t) / \mu-t| \\
& \leq \sup _{0 \leq t<(1+\varepsilon) T}|S(t) / \mu-t|=o_{\text {a.s. }}\left(T^{1-\alpha / 2+\delta}\right) .
\end{aligned}
$$

On writing now

$$
\mu t-\mu N(\mu t)=(S(N(\mu t))-\mu N(\mu t))+(\mu t-S(N(\mu t))),
$$

via (19) and (39), for the first term of (41), as $T \rightarrow \infty$, we have uniformly in $t \in[0, T]$

$$
\begin{aligned}
& S(N(\mu t))-\mu N(\mu t)=\frac{J_{1} k_{\alpha}}{\sigma} W_{1-\alpha / 2}(N(\mu t))+o_{\text {a.s. }}\left(T^{\gamma / 2+\delta}\right) \\
& =\frac{J_{1} k_{\alpha}}{\sigma} W_{1-\alpha / 2}(t)+\frac{J_{1} k_{\alpha}}{\sigma}\left(W_{1-\alpha / 2}(N(\mu t))-W_{1-\alpha / 2}(t)\right)+o_{\text {a.s. }}\left(T^{\gamma / 2+\delta}\right) .
\end{aligned}
$$

Hence, it suffices to bound the increments

$$
\sup _{0 \leq t \leq T}\left|W_{1-\alpha / 2}(N(\mu t))-W_{1-\alpha / 2}(t)\right|
$$

for large $T$. For doing this, we make use of a result of Ortega [23] as quoted in (27) of Remark 2.9.

On using now (27) in combination with (39), as $T \rightarrow \infty$, we arrive at

$$
\begin{aligned}
& \sup _{0 \leq t \leq T}\left|W_{1-\alpha / 2}(N(\mu t))-W_{1-\alpha / 2}(t)\right| \\
& \leq \sup _{0 \leq t \leq(1+\varepsilon) T} \sup _{0 \leq s \leq T^{1-\alpha / 2}}\left|W_{1-\alpha / 2}(t+s)-W_{1-\alpha / 2}(s)\right|=O_{\text {a.s. }}\left(T^{(1-\alpha / 2)^{2}+\delta}\right)
\end{aligned}
$$


with any $\delta>0$. Consequently, as $T \rightarrow \infty$, by (42), for the first term of (41), we conclude

$$
S(N(\mu t))-\mu N(\mu t)=\kappa_{\alpha} J_{1} W_{1-\alpha / 2}(t)+O_{\text {a.s. }}\left(T^{(1-\alpha / 2)^{2}+\delta}+T^{\gamma / 2+\delta}\right)
$$

uniformly in $t \in[0, T]$.

As to the second term of (41), on putting $n=N(\mu t)$, we have $S(n) \leq \mu t \leq$ $S(n+1)$. Consequently, via (39), as $T \rightarrow \infty$, we have uniformly in $t \in[0, T]$

$$
|S(N(\mu t))-\mu t| \leq \sup _{1 \leq k \leq N(\mu t)}|S(k+1)-S(k)| \leq \sup _{1 \leq k \leq(1+\varepsilon) T}|S(k+1)-S(k)|
$$

with any $\varepsilon>0$. On estimating now the term on the right-hand side of the last inequality in (44) via (19), by (44) and using also (27) with $a_{T}=1$, as $T \rightarrow \infty$, we obtain

$$
\begin{gathered}
\left.\sup _{0 \leq t \leq T} \mid S(N(\mu t))-\mu t\right) \mid \\
\leq \frac{J_{1} \kappa_{\alpha}}{\sigma} \sup _{0 \leq s \leq(1+\varepsilon) T}\left|W_{1-\alpha / 2}(s+1)-W_{1-\alpha / 2}(s)\right|+o_{\text {a.s. }}\left(T^{\gamma / 2+\delta}\right)=o_{\text {a.s. }}\left(T^{\gamma / 2+\delta}\right),
\end{gathered}
$$

with any $\delta>0$.

On putting now together (45), (44), (41), the proof of Theorem 2.2 is seen to be complete.

\subsection{Proof of Theorem 2.3}

Assume the conditions of Theorem 2.3, i.e., those of Theorem 2.1 and condition (iii). We note in passing that condition (iii) implies that of (i) in view of our assumption throughout that $J_{1}=\mathrm{E}\left(G\left(\tilde{\eta}_{0}\right) \tilde{\eta}_{0}\right) \neq 0$, for the sake of having the Hermite rank of $G$ to be 1 .

To begin with, we note that the algebraic identity of (2.5) in [3] continues to hold true in our present context. Consequently, with $S(t)$ and $Q(t)$ as in (12) and (14) respectively, for $Z(t)$ as in (15), we have the following identity:

$$
Z(t)=\frac{1}{2}(S(t)-\mu t)^{2}+A(t)-\frac{1}{2} Q^{2}(t)
$$

where

$$
A(t)=\mu \int_{N(\mu t)}^{t}(S(s)-\mu s-(S(t)-\mu t)) d s .
$$

In view of Theorem 2.1 and (40), when estimating $A(t)$, we arrive at

$$
A(t)=\frac{\mu \kappa_{\alpha} J_{1}}{\sigma} \int_{N(\mu t)}^{t}\left(W_{1-\alpha / 2}(s)-W_{1-\alpha / 2}(t)\right) d s+o_{\text {a.s. }}\left(T^{1-\alpha / 2+\gamma / 2+\delta}\right) .
$$


For estimating the integral in the latter conclusion, we have

$$
\begin{gathered}
\left|\int_{N(\mu t)}^{t}\left(W_{1-\alpha / 2}(s)-W_{1-\alpha / 2}(t)\right) d s\right| \\
\leq|N(\mu t)-t| \sup _{0 \leq t \leq T} \sup _{0 \leq u \leq T^{1-\alpha / 2+\delta}}\left|W_{1-\alpha / 2}(t+u)-W_{1-\alpha / 2}(t)\right| \\
=o_{\text {a.s. }}\left(T^{1-\alpha / 2+(1-\alpha / 2)^{2}+\delta}\right),
\end{gathered}
$$

as $T \rightarrow \infty$, where we used (31) with $a_{T}=T$ in Corollary 2.12, as well as (27) with $a_{T}=T^{1-\alpha / 2}$ in Remark 2.9. Thus, in view of (47) and (48), as $T \rightarrow \infty$, we arrive at

$$
\sup _{0 \leq t \leq T}|A(t)|=o_{\text {a.s. }}\left(T^{2-3 \alpha / 2+\alpha^{2} / 4+\delta}+T^{1-\alpha / 2+\gamma / 2+\delta}\right) .
$$

Next, in order to estimate $Q^{2}(t)$ in the identity (46), we have (cf. (14))

$$
\begin{gathered}
Q(t)=S(t)-\mu t-(S(N(\mu t))-\mu N(\mu t))-(\mu t-S(N(\mu t)) \\
=\frac{J_{1} \kappa_{\alpha}}{\sigma} W_{1-\alpha / 2}(t)+o_{\text {a.s. }}\left(t^{\gamma / 2+\delta}\right) \\
-\left(\frac{J_{1} \kappa_{\alpha}}{\sigma} W_{1-\alpha / 2}(t)+o_{\text {a.s. }}\left(t^{\gamma / 2+\delta}\right)+o_{\text {a.s. }}\left(t^{(1-\alpha / 2)^{2}+\delta}\right)\right) \\
+o_{\text {a.s. }}\left(t^{\gamma / 2+\delta}\right),
\end{gathered}
$$

where, as $t \rightarrow \infty$, we made respective use of (19) of Theorem 2.1, (43) and (45). Consequently, as $T \rightarrow \infty$, we arrive at

$$
\sup _{0 \leq t \leq T} Q^{2}(t)=o_{\text {a.s. }}\left(T^{\gamma+\delta}+T^{2(1-\alpha / 2)^{2}+\delta}\right) .
$$

On combining now the identity of (46) with (49) and (50), we conclude Theorem 2.3 .

\section{References}

[1] R. R. Bahadur. A note on quantiles in large samples. Ann. Math. Statist., 37:577-580, 1966.

[2] E. Csáki, M. Csörgö and R. Kulik. On Vervaat processes for sums and renewals in weakly dependent cases. In Dependence in Probability, Analysis and Number Theory. A Volume in Memory of Walter Philipp. Berkes et al., ed., pages 145156. Kendrick Press, Heber City, UT, 2010. 
[3] E. Csáki, M. Csörgő, Z. Rychlik, and J. Steinebach. On Vervaat and Vervaaterror-type processes for partial sums and renewals. J. Statist. Plann. Inference, 137(3):953-966, 2007.

[4] M. Csörgó and R. Kulik. Reduction principles for quantile and Bahadur-Kiefer processes of long-range dependent linear sequences. Probab. Theory Related Fields, 142(3-4):339-366, 2008.

[5] M. Csörgő and R. Kulik. Weak convergence of Vervaat and Vervaat error processes of long-range dependent sequences. J. Theoret. Probab., 21(3):672$686,2008$.

[6] M. Csörgő and P. Révész. How big are the increments of a Wiener process? Ann. Probab. 7:731-737, 1979.

[7] M. Csörgő and P. Révész. Strong Approximations in Probability and Statistics. Academic Press, New York, 1981.

[8] M. Csörgő, B. Szyszkowicz, and L. Wang. Strong invariance principles for sequential Bahadur-Kiefer and Vervaat error processes of long-range dependent sequences. Ann. Statist., 34(2):1013-1044, 2006.

[9] M. Csörgő, B. Szyszkowicz, and L. Wang. Correction: "Strong invariance principles for sequential Bahadur-Kiefer and Vervaat error processes of longrange dependent sequences" [Ann. Statist. 34 (2006), no. 2, 1013-1044;]. Ann. Statist., 35(6):2815-2817, 2007.

[10] H. Dehling and M. S. Taqqu. The empirical process of some long-range dependent sequences with an application to $U$-statistics. Ann. Statist., 17(4):1767$1783,1989$.

[11] L. Horváth. Strong approximation of renewal processes. Stochastic Process. Appl., 18(1):127-138, 1984.

[12] L. Horváth. Strong approximations of renewal processes and their applications. Acta Math. Hungar., 47(1-2):13-28, 1986.

[13] J. Kiefer. On Bahadur's representation of sample quantiles. Ann. Math. Statist., 38:1323-1342, 1967.

[14] J. Kiefer. Deviations between the sample quantile process and the sample df. In Nonparametric Techniques in Statistical Inference (Proc. Sympos., Indiana Univ., Bloomington, Ind., 1969), pages 299-319. Cambridge Univ. Press, London, 1970. 
[15] J. Komlós, P. Major, and G. Tusnády. An approximation of partial sums of independent RV's and the sample DF. I. Z. Wahrscheinlichkeitstheorie und Verw. Gebiete, 32:111-131, 1975.

[16] J. Komlós, P. Major, and G. Tusnády. An approximation of partial sums of independent RV's, and the sample DF. II. Z. Wahrscheinlichkeitstheorie und Verw. Gebiete, 34(1):33-58, 1976.

[17] N. Kôno. Classical limit theorems for dependent random sequences having moment conditions. In Probability theory and mathematical statistics (Tbilisi, 1982), volume 1021 of Lecture Notes in Math., pages 315-319. Springer, Berlin, 1983.

[18] T. L. Lai and W. Stout. Limit theorems for sums of dependent random variables. Z. Wahrsch. Verw. Gebiete, 51(1):1-14, 1980.

[19] D. Monrad and H. Rootzén. Small values of Gaussian processes and functional laws of the iterated logarithm. Probab. Theory Related Fields, 101(2):173-192, 1995.

[20] H. Oodaira. On Strassen's version of the law of the iterated logarithm for Gaussian processes. Z. Wahrscheinlichkeitstheorie und Verw. Gebiete, 21:289299, 1972.

[21] H. Oodaira. Some limit theorems for the maximum of normalized sums of weakly dependent random variables. In Maruyama, G., and Prokhorov, J.V. (eds.) Proceedings of the Third Japan-USSR Symposium on Probability Theory. Lecture Notes in Math. Vol. 550, Springer, Berlin, Heidelberg, New York, pp. 467-474.

[22] S. Orey. Growth rate of certain Gaussian processes. In Proceedings of the Sixth Berkeley Symposium on Mathematical Statistics and Probability (Univ. California, Berkeley, Calif., 1970/1971), Vol. II: Probability theory, pages 443451, Berkeley, Calif., 1972. Univ. California Press.

[23] J. Ortega. On the size of the increments of nonstationary Gaussian processes. Stochastic Process. Appl., 18(1):47-56, 1984.

[24] M. S. Taqqu. Weak convergence to fractional Brownian motion and to the Rosenblatt process. Z. Wahrscheinlichkeitstheorie und Verw. Gebiete, 31:287302, 1974/75.

[25] M. S. Taqqu. Law of the iterated logarithm for sums of non-linear functions of Gaussian variables that exhibit a long range dependence. Z. Wahrscheinlichkeitstheorie und Verw. Gebiete, 40(3):203-238, 1977. 
[26] M. S. Taqqu. Fractional Brownian motion and long-range dependence. In Theory and applications of long-range dependence, pages 5-38. Birkhäuser Boston, Boston, MA, 2003.

[27] W. Vervaat. Success epochs in Bernoulli trials (with applications in number theory). Mathematisch Centrum, Amsterdam, 1972. Mathematical Centre Tracts, No. 42.

[28] W. Vervaat. Functional central limit theorems for processes with positive drift and their inverses. Z. Wahrscheinlichkeitstheorie und Verw. Gebiete, 23:245253, 1972.

[29] Q. Wang, Y-X. Lin, and C. M. Gulati. Strong approximation for long memory processes with applications. J. Theoret. Probab., 16(2):377-389, 2003. 